Article

\title{
Evaluation of the Transepidermal Penetration of a Carnosine Complex in Gel Formulation by 3D Skin Models
}

\author{
Valeria Dissette ${ }^{1}{ }^{(\mathbb{D}}$, Carlo Alberto Bignozzi $^{3}{ }^{-}$, Giuseppe Valacchi $^{2}$, Alessandra Pecorelli ${ }^{2}$, \\ Stefano Manfredini ${ }^{1, *(\mathbb{D})}$ and Silvia Vertuani ${ }^{1}$ \\ 1 Department of Life Sciences and Biotechnology, Master Course in Cosmetic Science and \\ Technology (COSMAST), University of Ferrara, Via L. Borsari 46, 44121 Ferrara, Italy; \\ valeria.dissette@unife.it (V.D.); vrs@unife.it (S.V.) \\ 2 Department of Animal Science, Plants for Human Health Institute, North Caroline State University, \\ Kannapolis, NC 28081, USA; gvalacc@ncsu.edu (G.V.); apecore@ncsu.edu (A.P.) \\ 3 Department of Chemistry and Pharmaceutical Sciences University of Ferrara Via Fossato di Mortara 17, \\ 44121 Ferrara, Italy; g4s@unife.it \\ * Correspondence: mv9@unife.it; Tel.: +39-0532455294
}

Received: 12 September 2018; Accepted: 3 November 2018; Published: 14 November 2018

\begin{abstract}
Carnosine has several physiological roles, from intracellular $\mathrm{pH}$ buffering to antioxidant activities, which all depend on bioavailability. This study was conducted in a human skin 3D model and focuses on the effects of the topical delivery of carnosine, from a dermo-cosmetic gel, through the stratum corneum in the presence of a magnesium ion as a complexing agent. To evaluate possible enhancement for small peptide delivery to the skin from simple cosmetic formulations, we discovered that complexation was able to improve the delivery of carnosine into human skin 3D models by application in gel formulation. The concentrations of carnosine released in the underlying media and those that remained in the reconstructed human epidermis (RHE) tissues after 24 and $48 \mathrm{~h}$ exposure were measured. Moreover, the influence of magnesium ions was also evaluated comparing the same formulation with and without the salt. The results obtained in this study support hypothesis that magnesium can influence the delivery of small peptides and that the gel formulation based on the carnosine-magnesium complex allows for superior delivery of carnosine in the lower skin layer at a concentration up to $60 \%$ more than carnosine alone.
\end{abstract}

Keywords: carnosine; complexation; dermo-cosmetic formulation; topical delivery

\section{Introduction}

The efficacy of any cosmetic product containing an active ingredient is determined by two factors: The intrinsic activity of the molecule and the delivery of this molecule to its site of action. The intrinsic activity of an active ingredient, or its capability to express efficacy, dictates its functional profile but does not guarantee the efficacy of the final formulation containing the ingredient.

To express its function, the active ingredient must be delivered to the site of action at the right concentrations and the right time. This criterion applies to all types of active ingredients with any functionality and is of particular importance in dermo-cosmetic science [1].

Because the skin is the most extended organ of the human body, these concepts are particularly relevant due to the large surface for application. However, to reach effective concentrations in the cutaneous tissues deeper layers, the uppermost barrier, the stratum corneum (SC), must be overcome. In addition to specific transport mechanisms, due to the lipophilic properties of the SC, hydrophilic 
molecules penetrate poorly. Therefore, achieving skin bioavailability of hydrophilic active substances, such as peptides or peptide-like substances, is challenging [2].

The focus of the present study was to investigate the delivery of a simple dipeptide, L-carnosine, by topical application to human skin by means of a gel formulation containing L-carnosine in association with a magnesium ion, thus forming a carnosine complex. The aim of this complex is to improve the bioavailability by acting as a multifunctional ingredient with several roles, including antioxidant effects, buffering enzyme and sarcoplasmic reticulum calcium $\left(\mathrm{Ca}^{2+}\right)$ regulations [3].

Carnosine ( $\beta$-alanyl-L-histidine) is a dipeptide with a molecular weight of $226.23 \mathrm{Da}$ and is very hydrophilic, with a partition coefficient $(\log \mathrm{P})$ of $-2.972 \pm 0.436$ [4]. As a component of several tissues, it has several biological roles, including $\mathrm{pH}$ buffering and excellent antioxidant properties [5]. Carnosine is based on an indole nucleus (histidine-containing), particularly present in skeletal muscle [6].

The degrading enzyme, carnosinase, is also present in most tissues in the body, excepting skeletal muscle. This may explain why carnosine concentrations are highest in this tissue.

In human skeletal muscle, the levels of carnosine ranges between $5-10 \mathrm{mM}$ wet weight or $15-40 \mathrm{mmol} / \mathrm{kg}$ dry weight. Depending on the muscle mass these concentrations differ among animal species [7].

Slow-twitch muscle fibers contain low carnosine level, animals that are characterized by prolonged hypoxic dives, frequent sprints, and explosive flight behaviors have higher initial concentrations [7-9]. This fact is also confirmed in anaerobic sports (i.e., higher intramuscular concentrations of carnosine) [8-10].

On the other side, carnosine supplementation does not increase plasma levels due to the high activity of carnosinase. Only $14 \%$ of the ingested carnosine was found in urine and because $\beta$-ALA and L-histidine are the precursors of carnosine, the research pointed towards their supplementation [11].

Moreover, carnosine displays other physiological roles, including protection against oxidative stress. This latter is due to different mechanisms such as: Metal ion chelation, scavenging reactive oxygen species (ROS) and peroxyl radicals [8]. ROS can arise from exercise in several proposed mechanisms, i.e., increased flow of electrons in the electron transport system, decrease in $\mathrm{pH}$ or increased respiration, all leading to oxygen release from hemoglobin with increase in $\mathrm{pO} 2$ in the tissues [12]. Carnosine is involved in anti-aging processes, as it seems to influence the cellular lifespan and onset of age-related change with an apparent rejuvenation of senescent cells. Even if the mechanism of action of carnosine is not yet clear, in vitro and in vivo studies confirm the positive effects for skin and muscles in the presence of carnosine as a supplementation as well as in topical applications [13]. A large body of literature has dealt with the intriguing properties of this small dipeptide, here following a few examples that support our interest toward this molecule for antiaging application.

The accumulation of advanced glycation end products (AGE) in the skin has been associated with skin aging, inhibition of glycation of extracellular matrix proteins can therefore help the structure and appearance of the skin. Carnosine has demonstrated anti-glycation activity when applied topically in ex vivo human skin explants. In this study, a facial cream showed a significant superior anti-glycation effect compared to aqueous carnosine solution (approximately double), thus indicating the importance of the vehicle [14]. In another study [15] the effect of carnosine in combination with urea and arginine, was evaluated against severe xerosis of the foot skin in patients with diabetes (a disease associated with accelerated ageing). The study continued for 8 months in patients with type 2 diabetes, with severe plantar xerosis, with an emollient glycerin-based cream (SEC). The use of a cream based on urea, arginine and carnosine increased skin hydration and improved skin dryness in type 2 diabetic patients compared to a commonly used emollient cream based on glycerol, with a greater effectiveness observed as early as 4 weeks after treatment.

In another study, the application of a low molecular weight peptides such as carnosine in geronto-cosmetology has been investigated in skin cell cultures of young and old rats, demonstrating, together with other small peptides, to stimulate the proliferation of skin fibroblasts by $29-45 \%$ [16]. 
This effect was observed at lower concentrations and levels during skin aging in old cell cultures than in young cell cultures. These data open up prospects for a new approach to the creation of cosmetological substances at the base of small peptides such as carnosine.

Exposure to ultraviolet rays (UVR) is an important risk factor for skin aging and the development of non-melanoma skin cancers (NMSC). Although traditional sunscreens remain the pillar for preventing UVR-induced skin damage, they cannot provide complete protection against the full spectrum of molecular lesions associated with UVR exposure. The formation of pyrimidine cyclobutane dimers (CPD), as well as oxidative damage to the bases of DNA, including the formation of 8-oxo-7,8-dihydro-2'-deoxyguanosin (8OHdG) are among the key DNA lesions associated with photoaging and tumorigenesis. In addition to DNA lesions, UVR-induced free radical formation can lead to protein carbonylation (PC), an important form of irreversible protein damage that inactivates their biological function. In a recent study [17] a new topical product composed of traditional sun filters, DNA repair enzymes and carnosine was compared with traditional commercial products based on sun filters, demonstrating, by comparative study, on irradiated skin biopsies, clear improvements in the oxidation markers used in the study, CPD, $8 \mathrm{OHdG}$ and PC. Thus reducing the risk of skin aging and NMSC.

It has been documented that cellular senescence associated with telomeres may contribute to certain age-related disorders, including increased incidence of cancer, wrinkles and decreased skin elasticity. It has been proposed that telomere length may not be a strong survival biomarker in older individuals, but it may be an informative biomarker of healthy aging. Natural compounds based on imidazole dipeptide carnosine may make it clinically possible to demonstrate that slowing down the rate of telomere shortening could slow down the human aging process in specific tissues where it is known that telomere shortening appears to be a distinctive sign of oxidative stress and disease. Preliminary longitudinal studies of elderly individuals, conducted in a recent study, suggest that longer telomeres associated with improved survival and oral nutritional support with non-hydrolysed carnosine are useful therapeutic tools for critical telomere length maintenance that can fundamentally be applied in prolonging life expectancy, increasing survival and the chronological age of an organism [18].

Finally, since the 2000s, peptides are used in topical formulation for collagen stimulation, wound healing, "Botox-like" wrinkle smoothing, as well as for antioxidative and antimicrobial effects. Particularly, carnosine and N-acetylcarnosine alone in a water solution are able to reduce UVB erythema in human skin; both peptides showed antioxidant capacity, with a higher significance in conjunction with vehicles improving the substances' skin penetration capabilities [19].

Many future research opportunities are offered in view of these different physiological roles, in particular investigating its role to improve exercise performance and/or reduce muscular fatigue. It will also be important to investigate the role of different nutritional approaches in increasing carnosine levels in the muscle to optimize physiological activity and/or exercise capacity.

Goebel et al. [2] investigated the penetration of carnosine through the SC in association with a penetration enhancer, such as pentylene glycol, in a hydrophilic vehicle, achieving a low concentration of the dipeptide in the SC. Taking this into account, the aim of our study, although preliminary, was to evaluate whether a strategy involving the complexation of carnosine can influence its bioavailability through the SC. As the complexing agent we selected the magnesium ion; its concentration is generally high in the SC but constantly low in all other layers [20], suggesting that the magnesium ion plays a role in epidermal functions, such as barrier homeostasis and epidermal differentiation [21].

\section{Materials and Methods}

\subsection{Materials}

All tested samples were from Pavia Farmaceutici s.r.l. (Copiano (PV), Italy). 
The study considered three different samples as hydrogel formulations with the aim of evaluating the role of the complexing agent, magnesium alone, as a penetration enhancer of carnosine.

The different formulations tested are summarized in Table 1.

Table 1. Sample A: Magnesium-carnosine complex in the vehicle; Sample B: Free carnosine, without the complexing agent, in the same vehicle; Sample C: Magnesium salt in the same vehicle.

\begin{tabular}{cccc}
\hline Product & Sample A & Sample B & Sample C \\
\hline Vehicle and L-Carnosine & $\mathrm{x}$ & $\mathrm{x}$ & \\
Vehicle and Magnesium & $\mathrm{x}$ & & $\mathrm{x}$ \\
Salt & $\mathrm{x}$ & $\mathrm{x}$ & $\mathrm{x}$ \\
Vehicle & &
\end{tabular}

The hydrogel termed as Sample A was designed to contain carnosine, magnesium sulfate, ethoxydiglycol, phenoxyethanol, ethylhexyl glycerin, sclerotium gum, glycerin and water. All the raw materials used for the preparation of the samples are compliant with Regulation 1223/2009 on cosmetic products.

Samples B and C were formulated with the same components with the exception of magnesium for the former and carnosine for the latter.

The concentration of magnesium salt in samples $\mathrm{A}$ and $\mathrm{C}$ was $1.64 \%$, while the concentration of L-carnosine in samples A and B was $1.50 \%$.

Sample A was prepared by adding all the ingredients to the water solution, at room temperature under stirring; the gelling agent sclerotium gum, at a concentration of $0.8 \%$, was added as the last ingredient. The solution was maintained under stirrer mixing, until the complete hydration of the polymer and gelation process was complete.

The samples appear as transparent and colorless gels, pH $5.5 \pm 1.0$, density $0.90 \pm 0.10 \mathrm{~g} / \mathrm{mL}$, viscosity (Brooksfield RV, Brooksfield-Urai Spa, Assago (Milan), Italy), spindle 6, rpm 10) 2500-4500 cps.

Sample A was stated as a cosmetic product. It was dermatologically tested (Occlusive Patch Test), applied under occlusive conditions on the healthy skin of 20 volunteers, and it resulted as a non-irritant if applied on human skin. The microbiological stability of Sample A was tested via a Challenge test, that confirmed the preservative system used in the formulation was compliant with a 24-month shelf-life granted by the manufacturer Pavia Farmaceutici srl.

Samples B and C were used as models in this study and they were prepared with the same procedures and raw materials reported for sample A.

\subsection{EpiDermTM Reconstructed Human Epidermis (RHE) Model}

Reconstructed human epidermis (RHE) was used as a human skin tissue model. The EpiDermTM RHE model consists of a three-dimensional epidermal tissue grown at an air-liquid interface from normal human keratinocytes. The model is histologically similar to the human epidermis, presenting all differentiated cellular layers, and features a functional permeability barrier. The EpiDermTM Tissue Model was purchased from MatTek (MatTek In Vitro Life Science Laboratories, Bratislava, Slovak Republic).

Upon reception, tissues were transferred to SkinEthicTM Maintenance Medium and kept at $37^{\circ} \mathrm{C}$ in a humidified $5 \% \mathrm{CO}_{2}$ atmosphere until hydrogel application.

\subsection{Hydrogel Application on EpiDermTM RHE and Sample Collection}

Before the application of the three hydrogels, the medium was removed, and fresh medium was added. Then, $10 \mu \mathrm{L}$ of the three different hydrogels was topically applied on the surface of a set of RHEs in a single dose for $24 \mathrm{~h}$ or in a repeated application at $24 \mathrm{~h}$ for a total of $48 \mathrm{~h}$ in another set of RHEs. Control tissues were exposed to the vehicle medium alone. All exposed hydrogels and control samples were assayed in triplicate using three sets of RHE for each group. 
At each time point, tissue medium aliquots were collected and centrifuged at $1000 \times g$ for $20 \mathrm{~min}$ at $4{ }^{\circ} \mathrm{C}$ to remove insoluble impurities and tissue debris. The clear supernatants were stored at $-20{ }^{\circ} \mathrm{C}$ until analysis. After washing with PBS and snap-freezing in liquid nitrogen, the RHE tissues were homogenized in a glass beaker with $150 \mu \mathrm{L}$ of PBS on ice. The homogenates were then centrifuged at $5000 \times g$ for $5 \mathrm{~min}$ to obtain the supernatants, which were stored at $-20^{\circ} \mathrm{C}$ until analysis.

\subsection{ELISA for Carnosine}

Carnosine levels in the RHE culture media and homogenates collected at different time points ( 24 or $48 \mathrm{~h}$ ) were determined using a competitive ELISA kit (Elabscience Biotechnology Co., Ltd., Houston, TX, USA) according to the manufacturer's instructions. All of the samples were assayed in duplicate. A calibration curve was performed using carnosine as a standard. The optical absorbance was measured with a microplate reader at $450 \mathrm{~nm}$, and results are expressed as $\mathrm{ng} / \mathrm{mL}$. The lower limit of detection for carnosine was $8.438 \mathrm{ng} / \mathrm{mL}$.

\subsection{Statistical Analysis}

Means \pm standard deviations (SD) were calculated, and a Student's $t$ test was used for the evaluation of statistical significance. A $p$-value of $<0.05$ was considered significant.

\subsection{Tissue Treatment and Sample Collection}

Before the treatments, the medium was removed, and fresh medium was added. For the treatments, $10 \mu \mathrm{L}$ of the three different samples (abbreviated as A: Carnosine complex; B: Free carnosine, C: Magnesium salt) was topically applied over RHE in a single dose for $24 \mathrm{~h}$ or in a repeated application at $24 \mathrm{~h}$ for a total of $48 \mathrm{~h}$. Control tissue (CTRL) was exposed only to the medium alone. Because no differences between 24 and $48 \mathrm{~h}$ were observed in control tissues, the samples were pooled and presented as the control group. All mixtures and control samples were assayed in triplicate using three sets of RHEs for each group.

Tissue medium aliquots were collected after 24 and $48 \mathrm{~h}$ of treatment and centrifuged for $20 \mathrm{~min}$ to remove insoluble impurities and tissue debris at $1000 \times g$ at $4{ }^{\circ} \mathrm{C}$. The clear supernatants were stored at $-20{ }^{\circ} \mathrm{C}$ until analysis. After washing with PBS and snap-freezing in liquid nitrogen, the RHE tissues were homogenized in a glass beaker with $150 \mu \mathrm{L}$ of PBS on ice. The homogenates were then centrifuged for $5 \mathrm{~min}$ at $5000 \times \mathrm{g}$ to obtain the supernatants, which were stored at $-20^{\circ} \mathrm{C}$ until analysis.

\section{Results}

To evaluate whether the new gel formulation containing the carnosine complex (sample A) was able to improve the delivery of dipeptides into human skin by topical application, the concentrations of carnosine released in the underlying media and those that remained in the RHE tissues after 24 and $48 \mathrm{~h}$ exposure were measured. The bioavailability properties of analogue hydrogel formulations without the active ingredient (L-carnosine) (sample C) or containing only free carnosine without magnesium ions (sample B) were also evaluated and compared to sample A.

\subsection{Carnosine Levels in Media}

At the first-time point, $24 \mathrm{~h}$ after the topical application, higher L-carnosine levels were detected in the medium from the RHE treated with the carnosine complex (sample A) compared to those measured in the media of control tissues ( $p=0.028$; Table 2 and Figure 1). In media from RHE treated with the formulation without enhancer (sample B), there was an increase in the carnosine level although it was not statistically significant (Figure 1) compared to those of the control tissues. Of note, the comparison between samples A and B showed a significantly higher level of dipeptide in media from RHE treated with the carnosine complex compared to the level detected in the medium of sample B $(p=0.043$, 
Table 2 and Figure 1). In the medium of sample C (vehicle), the carnosine level was comparable to the concentration of carnosine recorded for the control tissue (Table 2 and Figure 1).

At the second-time point $(48 \mathrm{~h})$, after two repeated applications, the carnosine level in the medium of the sample A was lower than the level detected at the first-time point, although they were again greater than in the control tissue (Table 2 and Figure 1).

The carnosine levels in the media of samples B and C were similar to those detected at the first-time point (Table 2 and Figure 1).

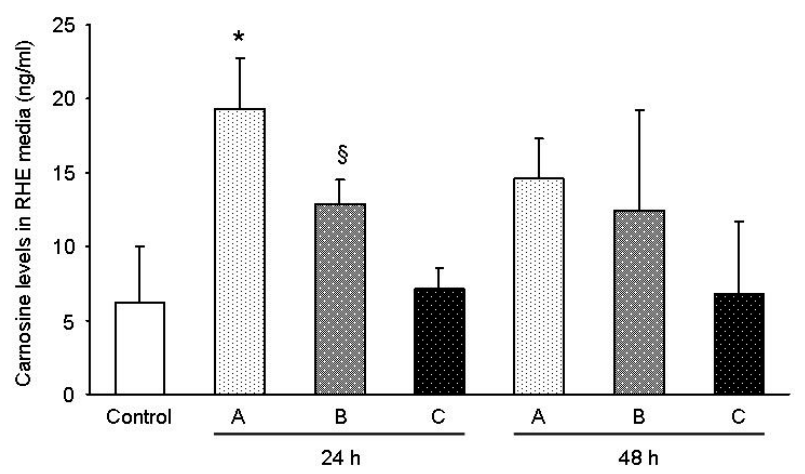

Figure 1. Carnosine levels in media from reconstructed human epidermis (RHE) tissues. Concentrations of carnosine released in media from RHE at 24 and $48 \mathrm{~h}$ after the topical application of different hydrogel formulations. A: Carnosine complex; B: Free carnosine; C: Magnesium salt in the same vehicle. Data are given as the means \pm standard deviations (SD) of three separate experiments. ${ }^{*} p<0.05$ versus control untreated RHE samples; $\S p<0.05$ versus sample A at $24 \mathrm{~h}$.

Table 2. Carnosine levels detected in media and lysates from RHE tissues treated with different gel formulations.

\begin{tabular}{ccccc}
\hline \multirow{2}{*}{ Time Points } & \multicolumn{2}{c}{ RHE Media } & RHE Lysates \\
\cline { 2 - 5 } & Group & Carnosine Levels $(\mathbf{n g} / \mathbf{m L})$ & Group & Carnosine Levels (ng/mL) \\
\hline & Control & $6.2 \pm 3.8$ & Control & $107.8 \pm 11.2$ \\
\hline \multirow{2}{*}{$1^{\wedge}$ time point } & Sample A & $19.3 \pm 3.4^{*}$ & Sample A & $65.9 \pm 9.4^{*}$ \\
$(24$ h) & Sample B & $12.9 \pm 1.6 \S$ & Sample B & $127.0 \pm 14.5+$ \\
& Sample C & $7.1 \pm 1.7$ & Sample C & $98.3 \pm 9.0$ \\
\hline \multirow{2}{*}{$2^{\wedge}$ time point } & Sample A & $14.6 \pm 2.7$ & Sample A & $98.0 \pm 8.1 \S$ \\
$(48$ h) & Sample B & $12.5 \pm 6.8$ & Sample B & $99.0 \pm 27.8$ \\
& Sample C & $6.7 \pm 5.0$ & Sample C & $105.6 \pm 7.3$ \\
\hline
\end{tabular}

Values are expressed as the means $\pm \mathrm{SD}^{*} p<0.05$ versus control tissue; $\S p<0.05$ versus Sample A at $24 \mathrm{~h}$;

$+p<0.01$ versus Sample A at $24 \mathrm{~h}$; Data from control tissues are obtained by the pooling of samples at 24 and $48 \mathrm{~h}$.

\subsection{Carnosine Levels in RHE Lysates}

To test the capacities of the different formulations to penetrate the skin, in the next step, we determined the carnosine levels in the RHE tissues.

After $24 \mathrm{~h}$, the tissues treated with sample A showed a carnosine content that was significantly lower than the level detected in the control tissue $(p=0.019$; Table 2 and Figure 2).

In the tissues treated with sample $B$, the carnosine level increased compared to the control tissues (Table 2 and Figure 2), but it was not statistically different $(p=0.708)$. Notably, the carnosine level in sample B was significantly higher than that in sample A ( $p=0.004$; Table 2 and Figure 2), indicating a tendency to retain carnosine. The carnosine level detected in the tissues treated with sample $\mathrm{C}$ was similar to the control (Table 2 and Figure 2).

After two repeated applications ( $48 \mathrm{~h}$ ), the carnosine levels detected were equivalent to those measured in the control tissues for all of the samples (Table 1 and Figure 2). 


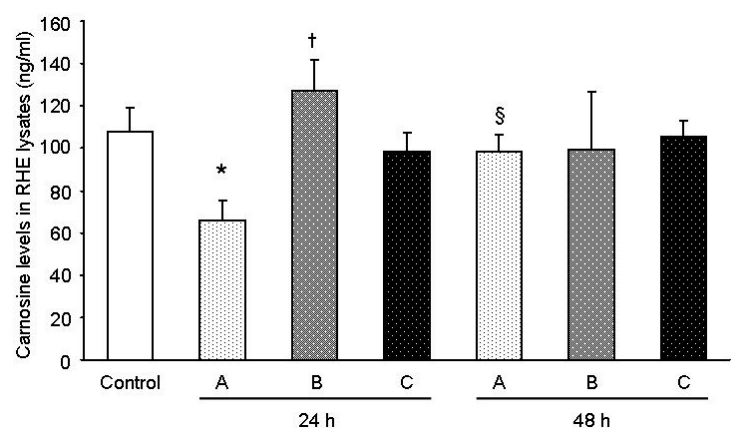

Figure 2. Carnosine levels in lysates from RHE tissues. Concentrations of carnosine retained in lysates of RHE at 24 and $48 \mathrm{~h}$ after the topical application of different hydrogel formulations. A: Carnosine complex; B: Free carnosine; C: Vehicle. Data are given as the means \pm SD of three separate experiments. ${ }^{*} p<0.05$ versus control untreated RHE samples; $+p<0.01$ versus sample A at $24 \mathrm{~h}$; $p<0.05$ versus sample $\mathrm{A}$ at $24 \mathrm{~h}$.

\section{Discussion}

The results obtained in this study support the view that the gel formulation based on the carnosine-magnesium complex allows for superior delivery of carnosine in the lower skin layer. According to the data, there was a $1.5 \times$ improvement in the carnosine concentration detected in the medium underneath the RHE layer following the application of sample A with respect to B, which only contained carnosine. This fact is consistent with the observation of a $1.9 \times$ residual quantity of carnosine on the surface of the RHE tissues for sample B with respect to A. As expected in sample C, the carnosine level was comparable to the concentration of carnosine recorded for the control tissue. Furthermore, recent studies [19] have already dealt with the issue related to the difficulties in delivering carnosine through diet and the necessity to develop an increased delivery on a tissue exposed to oxidation as skin, along with the possibility also to reach deeper layers. Schagen et al. [19] found an improvement using emulsions instead of solutions. In our case, the use of a chelating metal, greatly improved delivery in a simple formulation as a gel achieving better results. These preliminary results indicate that there are still a great possibility to ameliorate carnosine skin delivery by combination of metal chelation and better performing vehicles.

It is clear that an explanation of the higher topical availability of carnosine obtained in the presence of magnesium requires further investigation. However, we can advance the hypothesis that because carnosine is a polydentate ligand, it offers several potential binding sites: the two imidazole nitrogens, one carboxylic acid group and the amino groups. In aqueous solution, carnosine deprotonates the carboxylic function $(\mathrm{Pk}=2.6)$ [22] to form a negatively charged anionic species. Therefore, coordination with magnesium occurs through the interaction of the carboxylic function and the N1 nitrogen of the imidazole ring to form a 1:1 cationic carnosine-magnesium complex (Figure 3) with a stability constant on the order of 103. [23].

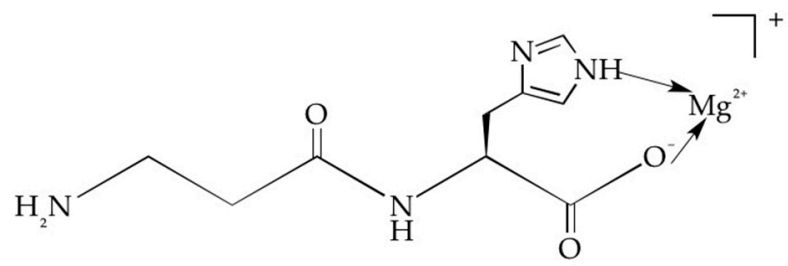

Figure 3. Schematic structure of the 1:1 carnosine-magnesium complex.

The positively charged complex in which carnosine is coordinated with the $\mathrm{Mg}^{2+}$ ion, which may be due to its small dimensions ( $86 \mathrm{pm}$ ), should experience an intense electric field and could therefore better interact with the electronic charges of the nitrogen atoms present on the membrane proteins and on the long chain glycosaminoglycans, thus favoring transmembrane transport of the amino acid. 


\section{Conclusions}

Our study, was conducted in a human skin 3D model represents a preliminary result. Despite that, the results obtained support the hypothesis that the complexation might be a strategy to improve the delivery of small polydentate peptides. Indeed, carnosine, selected by us for its interesting dermo cosmetic properties, resulted in successful delivery even by simple gel formulation. The carnosine-magnesium complex allows for superior delivery of carnosine in the lower skin layer up to $60 \%$ compared to the free carnosine. Further studies are currently ongoing to investigate combination of complexation with other forms of peptide skin delivery.

Author Contributions: V.D. and C.A.B. created the study design, G.V. and A.P. performed the measurements, G.V. and S.M. performed the data analyses, S.M. and S.V. designed the cosmetic formulation and drafted the manuscript, and all authors (V.D., C.A.B., G.V., A.P., S.M. and S.V.) participated in the discussion section and read and correct the final manuscript.

Funding: The study was supported by a post graduation grant from Ambrosialab s.r.l., Ferrara to the University of Ferrara (V.D. Grant 2016) and Pavia Farmaceutici s.r.l.

Acknowledgments: S.M. wish to thank Elisa Durini for kind assistance.

Conflicts of Interest: The authors declare no conflict of interest. The funders had no role in the design of the study; in the collection, analyses, or interpretation of data; in the writing of the manuscript, or in the decision to publish the results.

\section{References}

1. Wiechers, J.W. Skin Delivery: What it is and Why We Need it. In Science and Applications of Skin Delivery Systems; Allured Publ.: Carol Stream, IL, USA, 2008.

2. Goebel, A.S.B.; Schmaus, G.; Neubert, R.H.H.; Wohlrab, J. Dermal Peptide Delivery Using Enhancer Molecules and Colloidal Carrier Systems_Part I: Carnosine. Skin Pharmacol. Physiol. 2012, 25, 281-287. [CrossRef] [PubMed]

3. Begum, G.; Cunliffe, A.; Leveritt, M. Physiological Role of Carnosine in Contracting Muscle. Int. J. Sport Nutr. Exerc. Metab. 2005, 15, 493-514. [CrossRef] [PubMed]

4. SciFinderScholar: Advanced Chemistry Development [ACD/labs]. Software V8.14 for Solaris, TM 2007. Available online: https:/ / www.acdlabs.com/resources/freeware/index.php (accessed on 11 March 2015).

5. Babizhayev, M.A. Antioxidant activity of L-Carnosine, a natural histidine-containing dipeptide in crystalline lens. Biochim. Biophys. Acta 1989, 1004, 363-371. [CrossRef]

6. Culbertson, J.Y.; Kreider, R.B.; Greenwood, M.; Cooke, M. Effects of Beta-Alanine on Muscle Carnosine and Exercise Performance: A Review of the Current Literature. Nutrients 2010, 2, 75-98. [CrossRef] [PubMed]

7. Derave, W.; Ozdemir, M.S.; Harris, R.C.; Pottier, A.; Reyngoudt, H.; Koppo, K.; Wise, J.A.; Achten, E. Beta-alanine supplementation augments muscle carnosine content and attenuates fatigue during repeated isokinetic contraction bouts in trained sprinters. J. Appl. Physiol. 2007, 103, 1736-1743. [CrossRef] [PubMed]

8. Harris, R.C.; Marlin, D.J.; Dunnett, M.; Snow, D.H.; Hultman, E. Muscle buffering capacity and dipeptide content in the thoroughbred horse, greyhound dog and man. Comp. Biochem. Physiol. A Comp. Physiol. 1990, 97, 249-251. [CrossRef]

9. Suyama, Y.; Suzuki, T.; Maruyama, M.; Saito, K. Determination of carnosine, anserine and beta-alanine in the muscle of animals. Bull. Jpn. Soc. Sci. Fish. 1970, 36, 1048-1053. [CrossRef]

10. Hipkiss, A.; Brownson, C.; Bertani, M.; Ruiz, E.; Ferro, A. Reaction of carnosine with aged proteins: Another protective process? Ann. N. Y. Acad. Sci. 2002, 959, 285-294. [CrossRef] [PubMed]

11. Dunnett, M.; Harris, R.C. Influence of oral beta-alanine and L-histidine supplementation on the carnosine content of the gluteus medius. Equine Vet. J. Suppl. 1999, 30, 499-504. [CrossRef]

12. Boldyrev, A.A.; Dupin, A.M.; Bunin, A.Y.; Babizhaev, M.A.; Severin, S.E. The antioxidative properties of carnosine, a natural histidine containing dipeptide. Biochem. Int. 1987, 15, 1105-1113. [PubMed]

13. Hipkiss, A.R.; Baye, E.; de Courten, B. Carnosine and the processes of ageing. Maturitas 2016, 93, 28-33. [CrossRef] [PubMed] 
14. Narda, M.; Peno-Mazzarino, L.; Krutmann, J.; Trullas, C.; Granger, C. Novel facial cream containing carnosine inhibit formation of advanced glycation end-products in human skin. Skin Pharmacol. Physiol. 2018, 31, 324-331. [CrossRef] [PubMed]

15. Federici, A.; Federici, G.; Milani, M. Use of a urea, arginine and carnosine cream versus a standard emollient glycerol cream for treatment of severe xerosis of the feet in patients with type 2 diabetes: A randomized, 8 month, assessor-blinded, controlled trial. Curr. Med. Res. Opin. 2015, 31, 1063-1069. [CrossRef] [PubMed]

16. Chalisova, N.I.; Lin'kova, N.S.; Zhekalov, A.N.; Orlova, A.O.; Ryzhak, G.A.; Khavinson, V.K. Short peptides stimulate skin cell regeneration during ageing. Adv. Gerontol. 2014, 27, 699-703. [PubMed]

17. Emanuele, E.; Spencer, J.M.; Braun, M. An experimental double-blind irradiation study of a novel topical product (TPF 50) compared to other topical products with DNA repair enzymes, antioxidants, and growth factors with sunscreens: Implications for preventing skin aging and cancer. J. Drugs Dermatol. 2014, 13, 309-314. [PubMed]

18. Babizhayev, M.A.; Vishnyakova, K.S.; Yegorov, Y.E. Oxidative damage impact on aging and age-related diseases: Drug targeting of telomere attrition and dynamic telomerase activity flirting with imidazole-containing dipeptides. Recent Pat. Drug Deliv. Formul. 2014, 8, 163-192. [CrossRef] [PubMed]

19. Schagen, S.K. Topical Peptide Treatments with Effective Anti-Aging Results. Cosmetics 2017, 4, 16. [CrossRef]

20. Zglinicki, T.; Lindberg, M.; Roomans, G.M.; Forslind, B. Water and ion distribution profiles in human skin. Acta Derm. Venereol. 1993, 73, 340-343.

21. Denda, M.; Katagiri, C.; Hirao, T.; Maruyama, N.; Takahashi, M. Some magnesium salts and a mixture of magnesium and calcium salts accelerate skin barrier recovery. Arch. Dermatol. Res. 1999, 291, 560-563. [CrossRef] [PubMed]

22. Baran, E.J. Metal Complexes of Carnosine. Biochemistry 2000, 65, 789-797. [PubMed]

23. Lenz, G.R.; Martell, A.E. Metal Complexes of Carnosine. Biochemistry 1964, 3, 750-753. [CrossRef] [PubMed]

(C) 2018 by the authors. Licensee MDPI, Basel, Switzerland. This article is an open access article distributed under the terms and conditions of the Creative Commons Attribution (CC BY) license (http:/ / creativecommons.org/licenses/by/4.0/). 